\title{
Electrostatic charge distribution in armchair and zigzag carbon nanotubes: a numerical comparison of CNT charge models
}

Received: 13 November 2020 / Revised: 17 June 2021 / Accepted: 17 August 2021 / Published online: 11 November 2021 (C) The Author(s) 2021

\begin{abstract}
In engineering-especially in mechanics, lightweight engineering, aerospace engineering, electrical engineering as well as bioengineering - there is a desire of developing materials enabling an excellent performance with respect to mechanical, thermal, and electrical properties. One of the most promising materials are carbon nanotubes (CNTs), as they show excellent mechanical and electrical properties. To improve the understanding of the electrical behavior, i.e., the charge distribution in single-walled carbon nanotubes, both open ended armchair and zigzag types are investigated. In the present research, three different modeling approaches, a classic electrostatic model, the model by Li and Chou and the model by Mayer, are analyzed and compared with respect to their further applicability. In the numerical investigations, different test cases are performed: (i) the carbon nanotubes are charged with an overall charge, (ii) the CNTs are exposed to an external electric field, and (iii) the test cases (i) and (ii) are combined. Furthermore, the influence of different geometric parameters is investigated. It is shown that the charge applied to the CNTs distributes over the whole CNT having maxima at the ends of the tubes. These maxima can be influenced by both, the geometric parameters and the electric field strength.
\end{abstract}

\section{Introduction}

One of the highest driving factors of mankind is to be better, faster, and stronger. The same mindset applies to the materials humans are using and developing constantly. For that reason, a new structure formed out of carbon atoms was first discovered in 1952 by Radushkevich and Lukyanovich [1]. They discovered that carbon atoms not only can shape diamonds, graphene or fullerenes but also a tube, forming a carbon nanotube (CNT). Iijima [2] described later how this structure can be synthesized. This was the starting point for several investigations about this new and promising material. The different methods of fabrication were reported by $\mathrm{Lu}$ et al. [3], and the outstanding mechanical, thermal, and electrical properties are referred in [4-6].

As a result of different investigations about the mechanical and thermal properties, various applications have been proposed. For example, carbon nanotubes are applied in chemical sensors $[7,8]$ or for the detection of gases $[9,10]$.

One special feature of carbon nanotubes are their different conduction properties depending on the internal structure. CNTs can show metallic or semiconducting properties [11-13], depending on the orientation of the carbon atoms in the tube structure. Subsequently, CNTs are interesting, e.g., for nanotweezers [14,15], nanoscale actuators $[16,17]$ or transistors [18-20].

Despite of the numerous investigations, a large research effort is still necessary. Especially the reaction of a carbon nanotube to an applied voltage or an applied electric field is still of interest. Therefore, different

K. Lönnecke · O. Eberhardt · T. Wallmersperger ( $\varangle)$

TU Dresden, Institut für Festkörpermechanik, Professur für Mechanik multifunktionaler Strukturen, George-Bähr-Str. 3c, 01069 Dresden, Germany

E-mail: thomas.wallmersperger@tu-dresden.de 


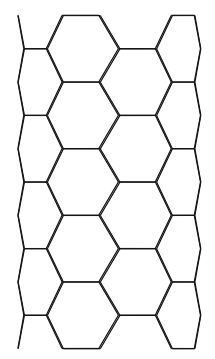

(a)

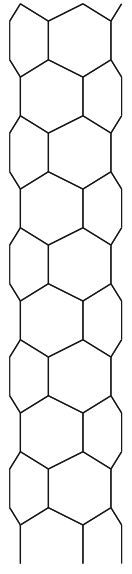

(b)

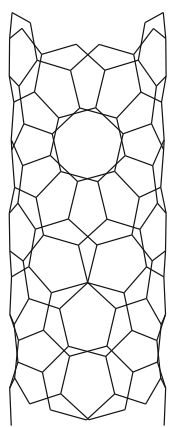

(c)

Fig. 1 a (5,5) armchair CNT (5 uc); b (5,0) zigzag CNT (5 uc); c (6,2) chiral CNT (1 uc)

models for simulating the charge distribution of CNTs have been proposed [21-24]. In the present research, the electrostatic behavior of carbon nanotubes is investigated by applying three different modeling approaches. In order to get a better understanding of the electric properties of carbon nanotubes, the modeling approaches are used to obtain results of the behavior of the nanotube toward (i) increasing overall charges and (ii) different applied electric fields. The motivation of the present investigation is to understand the developed modeling approaches, to compare them, and to get a first impression on the deformation of the CNTs, due to electromechanical coupling. Furthermore, it is interesting to understand the differences and decide which model can be preferred for which specific application. Additionally, the comparison will show if the modeling approaches are equally suitable for armchair nanotubes and zigzag CNTs. In conclusion, the presented electrical models are the first step toward the electromechanical coupling - i.e., the deformation of CNTs [25] due to an applied charge or an applied electric field—of carbon nanotubes.

\section{Fundamentals and modeling approaches for the charge distribution in carbon nanotubes}

In order to understand (i) the mechanical structure of the carbon nanotubes and (ii) the computation of the charge distribution, a general introduction to CNTs is given. A carbon nanotube is formed by carbon atoms which are arranged in a very specific way. To visualize the structure, the easiest way is to start with a sheet of graphene. Graphene is a single layer of carbon atoms arranged in hexagons. In this structure, each carbon atom is $\mathrm{sp}^{2}$-hybridized. This means that three $\sigma$ orbitals are arranged in a 2D structure having three $120^{\circ}$ angles. Therefore, each of these carbon atoms in the sheet of graphene has three covalent bonds to its neighboring atoms.

In order to obtain a carbon nanotube, the graphene layer has to be rolled up until a cylinder is formed. Therefore, it is called the rollup-model. This procedure describes a single-walled carbon nanotube, consisting of one layer of atoms. There also exist multi-walled CNTs which are made out of more than one layer.

Depending on the direction in which the graphene sheet is rolled up, different types of nanotubes can be categorized: (i) armchair, (ii) zigzag, and (iii) chiral nanotubes, see Fig. 1. To define the rollup direction of the graphene sheet, the chiral vector is used. For more details, the interested reader is, e.g., referred to Dresselhaus et al. [4]. To classify the type of the nanotube, an integer pair $(n, m)$, resembling the chiral vector, is used. For the armchair configuration, $n$ and $m$ have the same value; for $m=0$, a zigzag tube is formed; all other combinations are called chiral nanotubes. The higher the values of $n$ and/or $m$ are, the larger is the diameter of the nanotube. The height or length of the nanotube is defined by the quantity of unit cells stacked together. A unit cell (uc) is the basic construction element of the structure.

In the present research, the polyhedral model according to Cox and Hill [26], which is more sophisticated and accurate than the rollup-model, is applied.

After giving a short introduction of the geometry of a carbon nanotube, the electrical properties will be investigated more detailed. Therefore, the charge distribution of the tube has to be calculated. Various models 
investigating this topic can be found in the literature, e.g., electrostatic capacitance calculations by Rotkin et al. [27,28], ab initio calculations based on a density functional theory by Keblinski et al. [21] or energy-based methods by Ke et al. [29].

In the present research, three models are presented due to (i) their relative low computational effort, (ii) their good possibility to be calibrated with experimental results, and (iii) the ability to be easily compared to each other. In section 2.1, the fundamental electrostatic model [21] is presented. Based on this, an approach by $\mathrm{Li}$ and Chou [22,30] is discussed in Sect. 2.2. In Sect. 2.3, the model by Mayer [24] is outlined.

\subsection{Classic electrostatic model}

This Section shortly discusses the origin of the models applied in the present paper. The more advanced versions of the charge models are referred to the work by Keblinski et al. [21]. They used a density functional calculation and local density approximation (LDA) for their simulations. These results are compared with a classical electrostatic approach. More detailed information about the procedure can be found in their publication [21].

By the time this the work was published, the experimental distance between two carbon atoms with a sp ${ }^{2}$ hybridization was recorded approximately as $d_{c-c}=1.2 \AA$; but Keblinski et al. [21] calculated the distance in their research as $d_{c-c}=1.31 \AA$.

More recent investigations show that the $\mathrm{C}-\mathrm{C}$ bond length is larger than previously calculated. In the works by Eberhardt and Wallmersperger [31,32], the bond distance is assumed to be $d_{c-c}=1.421 \AA$. Therefore, in the present research the effect of the bond length on the charge distribution will be investigated.

In the publication by Keblinski et al. [21], the charge distribution of an $(5,5)$ open-ended armchair nanotube consisting of 110 atoms is calculated. In order to have a reference configuration, the same type of CNT is used in the present research as in Ref. [21].

To calculate the electrostatic potential $V^{* 1}$, the following ansatz is used:

$$
V^{*}\left(r_{i}\right)=A q_{i}+\sum_{j=1}^{N} \frac{q_{i}}{r_{i, j}} ; \quad i \neq j .
$$

Every carbon atom $i$ of the nanotube is surrounded by a spherically distributed charge, see Fig. 2b. $r_{i, j}$ describes the distance between the atoms $i$ and $j, q_{j}$ and $q_{i}$ are the charges of the atoms $j$ and $i$, respectively.

The first term in Eq. (1) represents the self potential of each atom. Keblinski [21] et al. fitted the parameter $A$ to a good accordance with the quantum calculations of $A=0.78 \AA^{-1}$. In the following, this approach will be referred to as the classical model.

\subsection{Electrostatic model by Li and Chou}

Li and Chou [22,33] used a different approach than the classical one. They applied an atomistic moment method to calculate the distribution of the charges along the carbon nanotube. The main difference to the classical model is the area in which the electrostatic charge around the nucleus of the carbon atoms is spread. In Section 2.1, the charge of each atom is distributed in a sphere around the center, see Fig. 2b. In the model by $\mathrm{Li}$ and $\mathrm{Chou}$, referred to as $\mathrm{Li}$ model, it is distributed within a triangle on the nanotube surface, see Fig. 2c.

Similar to the previous approach, a description of the electric potential $V\left(r_{i}\right)$ for each atomic position $r_{i}$ for each of the $N$ atoms in the nanotube can be found. This can be formulated as

$$
V\left(r_{i}\right)=\sum_{j=1}^{N} \frac{q_{j}}{4 \pi \epsilon_{0} r_{i, j}} ; \quad i \neq j,
$$

where $\epsilon_{0}$ is the permittivity of the free space, $q_{j}$ is the charge of the atom $j$, and $r_{i, j}$ again describes the distance between atom $i$ and $j$ in a tube consisting of $N$ atoms. These $N$ equations, one for each atom, can

\footnotetext{
${ }^{1}$ In order to obtain the electric potential, $V^{*}$ has to be divided by a parameter which has the dimension $\left[\frac{\mathrm{C}}{\mathrm{Vm}}\right]$.
} 


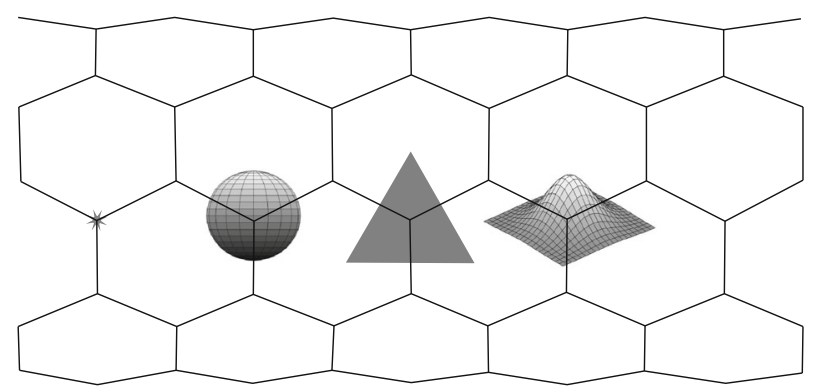

(a)

(b)

(c)

(d)

Fig. 2 a Point charge; b spherical charge; c triangular charge; $\mathbf{d}$ Gaussian charge distribution

be organized in a matrix form, see Eq. (3). The positions and therefore the distances are extracted from the polyhedral model derived by applying the molecular mechanical model by Cox and Hill [26],

$$
[A]\{q\}=\left\{V_{0}\right\} .
$$

Here, $\{q\}$ and $\left\{V_{0}\right\}$ denote the vector of the charge and the vector of the electric potential, respectively. The matrix [A] includes the interaction coefficients $a_{i j}$. In the case of two different atoms $i \neq j$, Eq. (2) shows that $a_{i j}$ can be expressed as

$$
a_{i j}=\frac{1}{4 \pi \epsilon_{0} r_{i, j}} .
$$

Please note that only the distance $r_{i, j}$ between two considered atoms $i$ and $j$ is relevant.

The following expression considers the self-potential of each atom, i.e., if $i$ equals $j$. Then, the triangle shape of the charge distributions becomes important. In this case, where $i=j$, the term for $a_{i j}$ results in

$$
a_{i i}=\frac{b \sqrt{3} \ln (2+\sqrt{3})}{4 \pi \epsilon_{0} s} .
$$

The term $s=\frac{3 \sqrt{3} b^{2}}{4}$ with $b=0.142 \mathrm{~nm}$ describes the triangular area in which the charge of the considered atom is distributed. A comparison of the model by Li and Chou and the model by Keblinski et al. [21] is performed in [22]. A good correlation between these two models is found. Therefore, in the present research, the newer and more sophisticated model by Li and Chou [22] is applied.

\subsection{Charge-Dipole model by Mayer}

In this Section, the model published by Mayer [24] is reviewed. In the following, it is referred as Mayer model. A short overview of this model is given in order to understand which parameters were used and how the solution procedure works.

This model describes every atom with a charge $q_{i}$ and also with a dipole $\mathbf{p}_{i}$. Subsequently, all calculations are performed with both, charges and dipoles. The consideration starts with the total energy $E_{\text {tot }}$ of all atoms in the nanotube at their given positions:

$E_{\text {tot }}=\frac{1}{2} \sum_{i=1}^{N} \sum_{j=1}^{N} q_{i} T_{q-q}^{i, j} q_{j}-\frac{1}{2} \sum_{i=1}^{N} \sum_{j=1}^{N} \mathbf{p}_{i} \cdot \mathbf{T}_{p-p}^{i, j} \mathbf{p}_{j}-\sum_{i=1}^{N} \sum_{j=1}^{N} \mathbf{p}_{i} \cdot \mathbf{T}_{p-q}^{i, j} q_{j}+\sum_{i=1}^{N} q_{i}\left(\chi_{i}+V_{i}\right)-\sum_{i=1}^{N} \mathbf{p}_{i} \cdot \mathbf{E}$.

Please note that Eq. (6) sums up the energy contributions of different interactions. The first term refers to the interaction between the charge of an atom and the charges of the other atoms. The sum is multiplied with $1 / 2$ 
to avoid the double counting of each pair of atoms. The term is weighted with the interaction coefficient $T_{q-q}^{i, j}$. This coefficient can be calculated with Eq. (7) for $i \neq j$,

$$
T_{q-q}^{i, j}=\frac{\frac{1}{4 \pi \epsilon_{0}} \operatorname{erf}\left(\frac{r_{i, j}}{\sqrt{2} R}\right)}{r_{i, j}} .
$$

Equation (7) looks similar to Eq. (4), which is considered for point charges. Mayer extended this approach and assumed that the charges are spread by a Gaussian distribution. The interaction coefficient $T_{q-q}^{i, j}$ is affected by the vacuum permittivity $\epsilon_{0}$ and the distance $r_{i, j}$ between atom $i$ and $j$ in a nanotube consisting of $N$ atoms. The term "erf()" denotes the error function, where $\operatorname{erf}(x)=\frac{2}{\sqrt{\pi}} \int_{0}^{x} e^{-\tau^{2}} d \tau$.

The most important parameter in Eq. (7) is the width of the Gaussian distribution $R$. If $R$ is small, the system reacts in a similar way as if the computation were performed with point charges, see Fig. 2a. If $R$ is larger, the charge located on each nucleus is distributed with a Gaussian curve, see Fig. 2d. This results in a more evenly distribution of the charges over the whole nanotube. At this point, it has to be mentioned that the used indices at the interaction terms are not tensor notations. $i$ and $j$ in this case just denote the atoms for which the interaction coefficient is computed, ()$_{q-q}$ denotes the interaction of two charges, ()$_{p-q}$ describes the interaction of a charge and a dipole, and ()$_{p-p}$ stands for the interaction of two dipoles.

For $i=j$, the self-energy coefficient is given by

$$
T_{q-q}^{i, i}=\frac{1}{4 \pi \epsilon_{0}}\left(\frac{\sqrt{\frac{2}{\pi}}}{R}\right) .
$$

The different contributions in Eq. (6) are explained now in more detail: The second term in Eq. (6) describes the interaction of two dipoles, and the third term represents the interaction of a charge and a dipole. This can be calculated by

$$
\mathbf{T}_{p-q}^{i, j}=\frac{1}{4 \pi \epsilon_{0}} \frac{\mathbf{r}_{i, j}}{r_{i, j}^{3}}\left[\operatorname{erf}\left(\frac{r_{i, j}}{\sqrt{2} R}\right)-\sqrt{\frac{2}{\pi}} \frac{r_{i, j}}{R} e^{\frac{-r_{i, j}^{2}}{2 R^{2}}}\right] ; \quad i \neq j
$$

for the second term and by

$$
\mathbf{T}_{p-p}^{i, j}=\frac{1}{4 \pi \epsilon_{0}}\left\{\frac{3 \mathbf{r}_{i, j} \otimes \mathbf{r}_{i, j}-r_{i, j}^{2} \mathbf{I}}{r_{i, j}^{5}}\left[\operatorname{erf}\left(\frac{r_{i, j}}{\sqrt{2} R}\right)-\sqrt{\frac{2}{\pi}} \frac{r_{i, j}}{R} e^{\frac{-r_{i, j}^{2}}{2 R^{2}}}\right]-\sqrt{\frac{2}{\pi}} \frac{1}{R^{3}} \frac{\mathbf{r}_{i, j} \otimes \mathbf{r}_{i, j}}{r_{i, j}^{2}} e^{\frac{-r_{i, j}^{2}}{2 R^{2}}}\right\} ; \quad i \neq j
$$

for the third term. $\mathbf{T}_{p-q}^{i, j}$ is a $(3 \times 1)$-vector, $\mathbf{T}_{p-p}^{i, j}$ is a $(3 \times 3)$-matrix. Please note that $\mathbf{r}_{i, j}$ is the vector from atom $i$ to atom $j$, and $\mathbf{I}$ represents the identity matrix. These terms can also formulated for $i=j$ by:

$$
\begin{aligned}
\mathbf{T}_{p-q}^{i, i} & =0, \\
\mathbf{T}_{p-p}^{i, i} & =-\frac{1}{4 \pi \epsilon_{0}} \frac{\sqrt{\frac{2}{\pi}}}{3 R^{3}} \mathbf{I} .
\end{aligned}
$$

The second to the last term in Eq. (6) for the total electrochemical energy describes the reaction of a charge to an applied external potential $V_{i}$. This is the energy which is required to hold or press the charge in the position given by the external potential. In the fourth term, the interaction of the additional charge $q_{i}$ with the electrons of the atom $i$ placed at the given position is accounted by $\chi_{i}$. In the publication by Mayer [24], $\chi_{i}$ is defined as the electron affinity. One year later, in 2008, in the research by Mayer and Åstrand [34], it is called electronegativity. On the Pauling scale this would mean $\chi_{i}=2.55$ for a carbon atom [35].

In the publication by Mulliken [36], it is shown that the electronegativity can be calculated by the ionization energy and the electron affinity. Unlike to the Pauling scale, this refers to an absolute electronegativity. Bultnick et al. [37] present a chart with different values for the electronegativity from $\chi=2.47$ to $\chi=8.58$, based on different possible calculation approaches.

Please note that the exact value of $\chi_{i}$ is nonrelevant as it cancels out if only one kind of atoms is considered. The last term of Eq. (6) defines the interaction of the dipoles and the applied external electric field $\mathbf{E}$. 


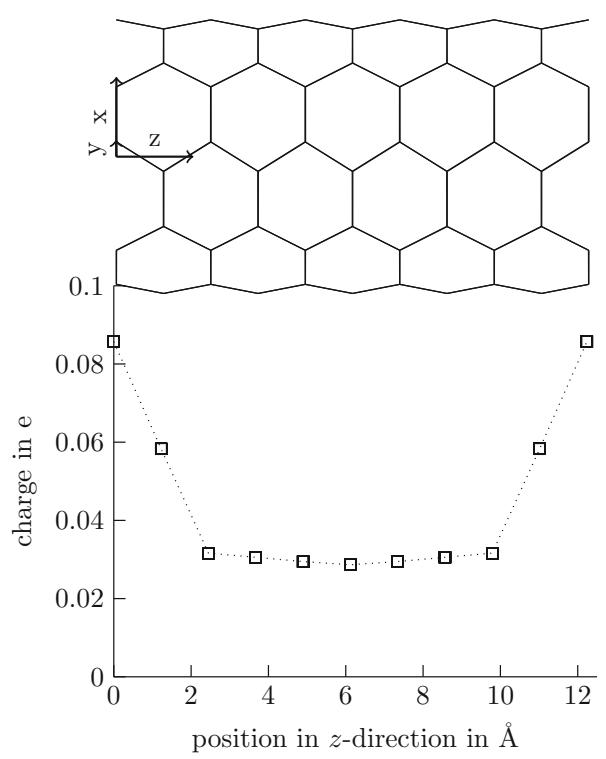

(a)
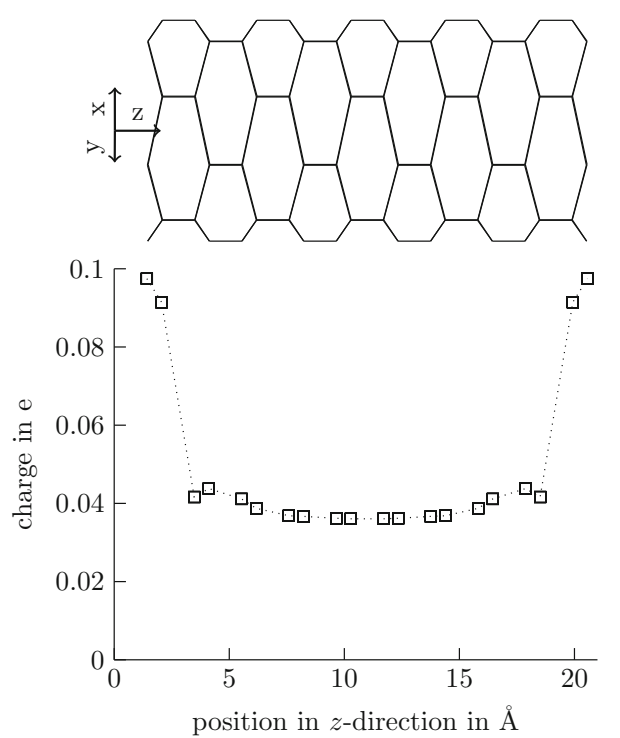

(b)

Fig. 3 a $(5,5)$ armchair CNT; b $(5,0)$ zigzag CNT with a total charge of 5e and 5 unit cells according to the model by Mayer

\section{Comparison of the different modeling approaches}

In order to show the details of the given models, different test cases are investigated. In the first test case, a carbon nanotube which is charged by an overall charge, i.e., a total electrical load, is considered. There, it can be observed how this total electrical load is distributed between the atoms of the carbon nanotube.

In Sect. 3.2, the second test case is formulated. In this case, the tube is exposed to an external electric field. In the last Section, the third test case will combine both effects, i.e., a charged carbon nanotube is exposed to an electric field.

\subsection{Test case I: overall charge}

In this first test case, a carbon nanotube is charged with an overall charge. At first the behavior of the CNT having an overall charge of $5 e$ is investigated, where $e=1.6022 \cdot 10^{-19} \mathrm{C}$ is the elementary electric charge. Afterward, different overall charges are compared in order to understand the electrical behavior of CNTs. Thereafter, the different models, mentioned in Sect. 2, are compared to analyze the differences. Both armchair and zigzag nanotubes are considered.

Figure 3 (top) shows two carbon nanotubes; in Fig. 3a the armchair $(n=5, m=5)$ configuration and in Fig. $3 \mathrm{~b}$ a zigzag $(n=5, m=0)$ one. The straight lines represent the covalent bonds between the carbon atoms. At each corner point, one carbon atom is located. Both constructs are axially symmetric with respect to the longitudinal $z$-axis. Therefore, in Fig. 3a behind each visible corner point there is another corner point. In total, the $(5,5)$ armchair nanotube consists of 110 atoms. The $(5,0)$ zigzag configuration consists of 100 atoms in total, see Fig. 3b. The atoms aligned vertically in Fig. 3 are called a row of atoms, as they show the same behavior with regard to the charge distribution.

Each of these CNTs has a length of five unit cells. One unit cell defines the basic construction element of a structure. Please note that for the zigzag CNT not exactly 5 unit cells are shown. In order to represent the symmetric behavior, the first row of atoms on the left side is omitted. The considered CNTs are open ended, i.e., they do not have any cap. In Fig. 3b, it can be seen that after two rows of atoms which are very close together the distance to the next row is slightly larger. The displayed results show that the charge distribution depends on the arrangement of the atoms in the nanotube.

Two graphs are depicted in Fig. 3 (bottom) directly underneath the tubes. Each of these graphs shows the charge in elementary electric charge $e$ with respect to the position of the atoms in the CNT in $\AA$. The data points are aligned with the atoms above. Atoms placed on the same position in $z$-direction have the same charge, due 


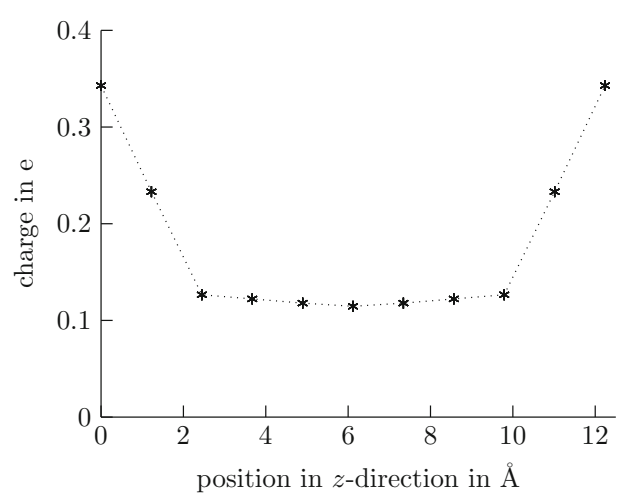

(a)

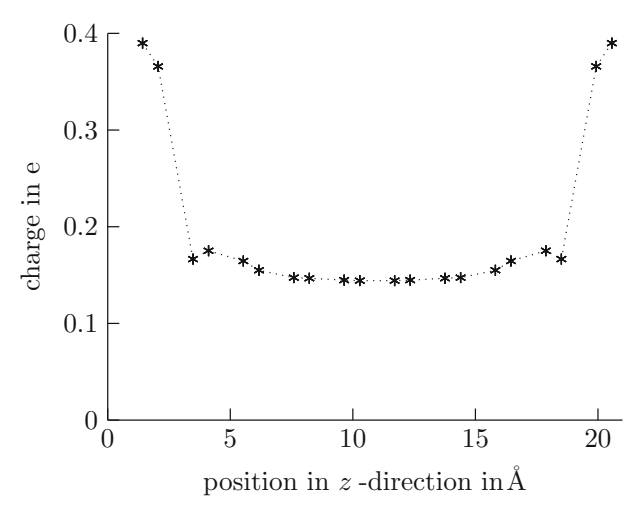

(b)

Fig. 4 a (5,5) armchair CNT; b $(5,0)$ zigzag CNT with a total charge of 20e and 5 unit cells according to the model by Mayer

to the rotational symmetry. Therefore, in Fig. 3a each data point represents 10 atoms (for an armchair CNT) and in Fig. $3 b 5$ atoms (for a zigzag CNT).

The nanotubes shown in Fig. 3 have an overall charge of 5 elementary charges. This total charge is distributed over the CNT as shown in the graph. Please note that, due to the distributed charges, also fractions of the elementary charge occur at certain local positions in the tube. The applied test case is just an exemplary case in order to demonstrate the general behavior. Therefore, we decided to use this first example as a fundamental example which can be extended to higher overall charges. In Fig. 3, it can be clearly seen that the atoms at the end of the tube have a higher charge compared to the average value. In the middle region of the nanotube, the charges are distributed almost evenly. The atoms of the considered nanotube are $\mathrm{sp}^{2}$-hybridized, i.e., each atom strives to be bonded to three other atoms. In the middle part of the nanotube, this criterion is fulfilled, i.e., all bindings are bounded. At both ends of the nanotube, due to the open end configuration, not all bindings are complete, i.e., there are free electrons on the carbon atoms which do not have all binding partners. The free electrons attract the additionally applied charge; this means that the charge is more concentrated at the ends of the nanotube. Please note that the total charge has been kept constant for the entire numerical simulation. If all charges are multiplied with the respective number of atoms and summed up, the result is exactly $5 \mathrm{e}$.

In the next investigation, an overall charge of 20e is applied, see Fig. 4. As in Fig. 3, it can be seen that the relative distribution of the charge is the same, i.e., it is independent of the total charge. If the overall charge increases while the total number of atoms remains constant, the charge on each atom has to be larger. For example: the value of the charge of the very last atoms on the tube (a) increases from $0.0857 \mathrm{e}$ in Fig. 3 to $4 \cdot 0.0857 \mathrm{e}=0.3429 \mathrm{e}$ in Fig. 4; for the ten atoms in the middle it increases from 0.0287e to 0.1146e.

It can be seen that the $(5,0)$ zigzag CNT shows small oscillations of the charge between neighboring atoms and the charge distribution is not as homogeneous as for the armchair nanotube. This can be justified with the value of the parameter $R$. A more detailed discussion of this will follow later on.

The correlation between higher overall charge and higher charges on each atom is depicted in Fig. 5. As shown in Figs. 3 and 4, again the charge distribution with respect to the longitudinal $z$-axis position of the atoms in the carbon nanotube is displayed. Five different curves are plotted in the graph: the same tube geometry is loaded with an overall charge of 1e, 2e, 5e, 10e, and 20e. As expected, it can be seen that with an increasing overall charge the charge of each atom increases. This behavior is independent of the tube configuration, i.e., whether it is an armchair tube or a zigzag one.

It can also be observed that the relative charge distribution between the ends and the middle of the tube increases with a higher prescribed overall charge.

As shown in Sect. 2, different approaches are used to represent the charge distribution on a carbon nanotube. Figure 6 gives the numerical results for a $(5,5)$ armchair and a $(5,0)$ zigzag CNT according to the model by $\mathrm{Li}$ and Chou. In the graph, the normalized charge over the length is given. Normalized charge means that the actual charge of the atom is divided by the average charge per atom $\bar{q}=\frac{q t o t}{N}$ where $\mathrm{N}$ is the number of atoms in the nanotube.

Figure 6a shows identical results compared to the work by Li and Chou [22]. This comparison was made to test the functionality of the programmed MATLAB application. 


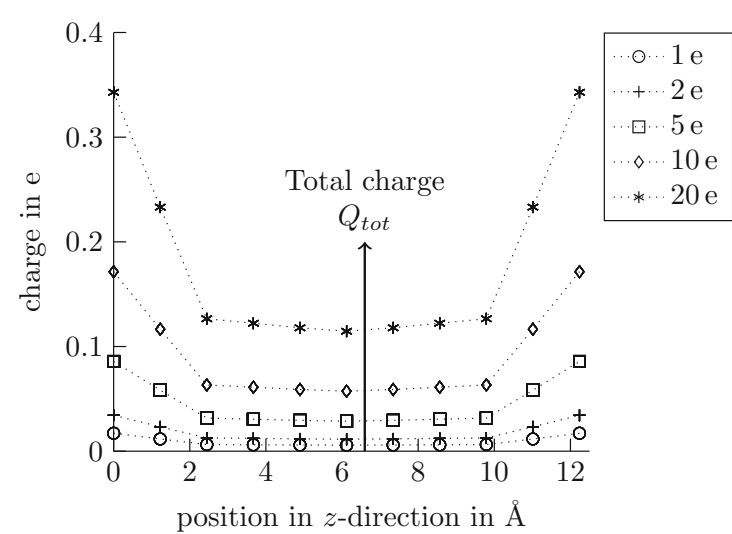

(a)

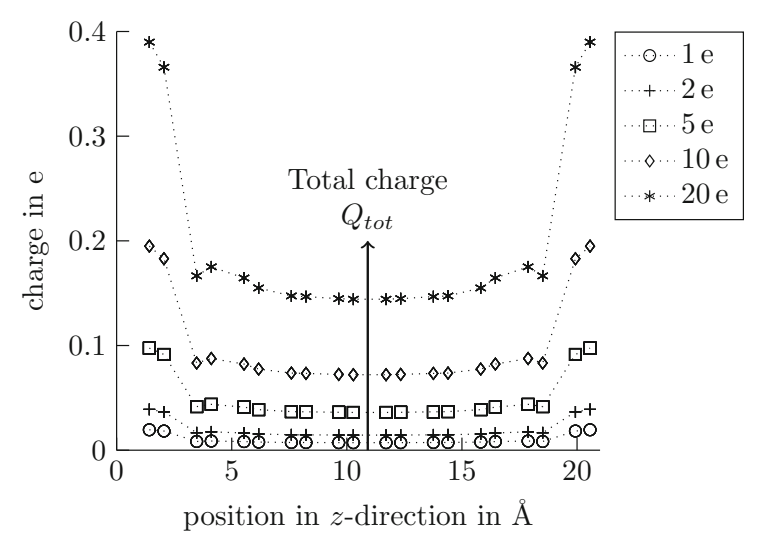

(b)

Fig. 5 Comparison of charges for different total charges of 1e, 2e, 5e, 10e, and 20e for a $(5,5)$ armchair CNTs and b $(5,0)$ zigzag CNTs having 5 unit cells according to the model by Mayer

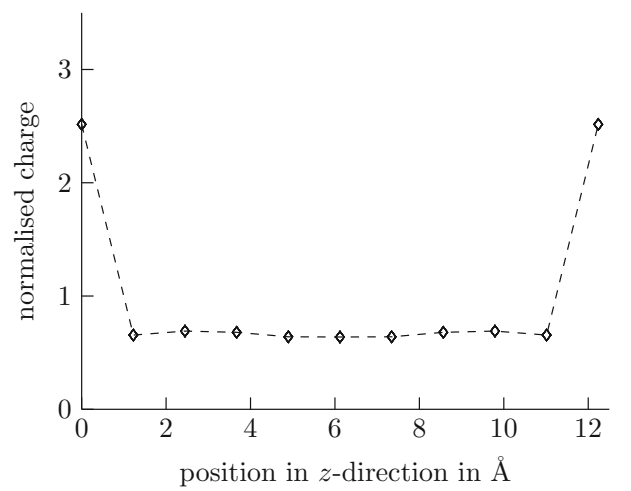

(a)

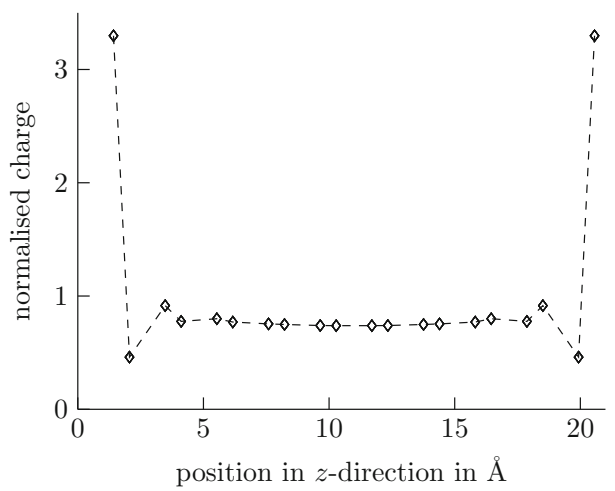

(b)

Fig. 6 a $(5,5)$ armchair CNT; $\mathbf{b}(5,0)$ zigzag CNT with normalized charge according to the model by Li an Chou. The tubes have an overall charge of $20 \mathrm{e}$ and a length of 5 unit cells

In order to compare the modeling approaches by $\mathrm{Li}$ and Chou and by Mayer, both results are shown in Fig. 7. For the armchair configuration as well as for the zigzag type, the charge distribution in the middle of the CNT is nearly the same for both modeling approaches.

At both ends of the nanotubes, a distinct difference can be observed. For both tube types, the charges of the last two rows of atoms show significant different values. This can be explained with the distribution approach of the charges at the nuclei in the different modeling approaches. As described in Sect. 2, the model by Li and Chou [22] distributes the charge over a triangular area. Mayer [24] on the other hand distributes the charge by using a Gaussian curve with the parameter $R$.

The approach by Mayer [24] seems to provide more homogeneous results. The increase in the charge of the last two rows of atoms in the armchair nanotube is less high than in the numerical simulation based on the model by Li and Chou. Also for zigzag nanotubes, the course of the charge seems to be more realistic. At both ends of the $(5,0)$ zigzag CNT, the last two rows of atoms are close together. The model by Li and Chou shows the highest charge value at the last atom row. For the middle part of the nanotube, the value decreases and increases again toward the end of the CNT. Nonetheless, the model by Mayer shows a more comprehensible trend. The last rows of atoms at the ends have the highest value, and the next row has a slightly lower value because of the small atom distance. Between the second row and third row, the decrease is higher due to the arrangement of the atoms in the nanotube.

Longer nanotubes show the same qualitative behavior as short nanotubes. Figure 8 gives the results of the same setup as Fig. 7, but with a different nanotube length of 20 unit cells. Likewise the values of the charge of the atoms in the middle of the nanotubes are the same for both models. The differences, shown in Fig. 8a, occur in the two rows of atoms facing both ends and in Fig. $8 \mathrm{~b}$ in the last four rows of atoms. This is a similar amount of atoms as in the investigations performed for shorter nanotubes. 


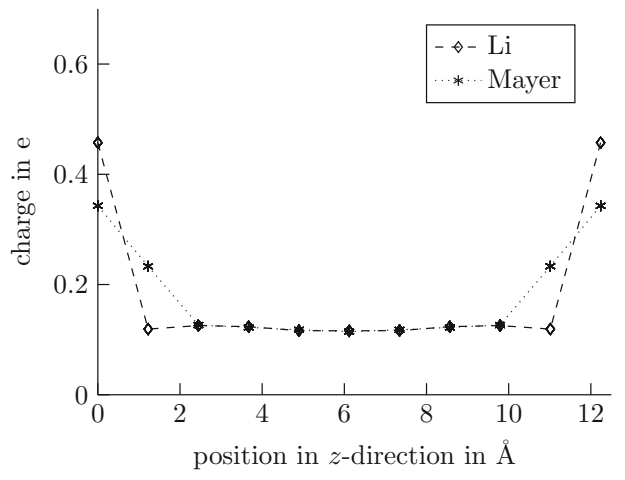

(a)

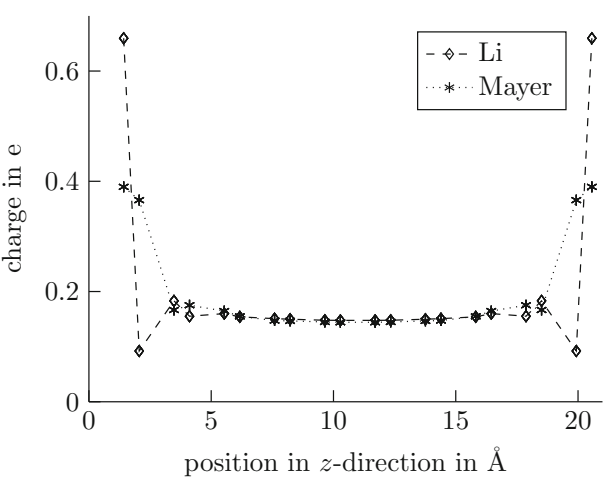

(b)

Fig. 7 a $(5,5)$ armchair CNT; $\mathbf{b}(5,0)$ zigzag CNT with a total charge of 20e and 5 unit cells. Comparison of the models by Li and Chou and by Mayer

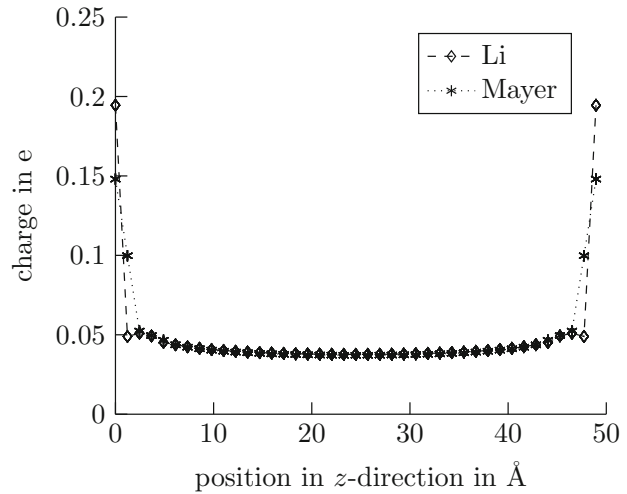

(a)

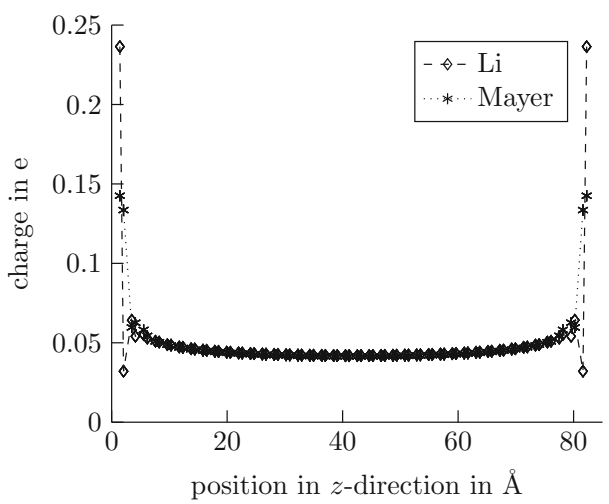

(b)

Fig. 8 a $(5,5)$ armchair CNT and b (5,0) zigzag CNT with a total charge of 20e and 20 unit cells. Comparison of the models by $\mathrm{Li}$ and Chou and by Mayer

The modeling approaches are therefore similar except of the charge accumulation at the ends of the tubes. Regardless of the length of the carbon nanotubes, only the last few rows of atoms are affected by the differences of the charge distribution.

In Fig. 9, the effects of different values of the parameter $R$, used in the Mayer model, are shown. Also the charge distribution with respect to the position of the atoms of the nanotube is given. The results for the $(5,5)$ armchair nanotubes are given in Fig. 9a and for the (5,0) zigzag nanotubes in Fig. 9b. The value of $R$, which shows the range in which the charge is located around the nucleus, is varied from $R=0.2 \AA$ to $R=0.9 \AA$.

The parameter $R$ is the decisive factor of the model by Mayer [24] in order to demonstrate how the charge on each atom is spread over the area around it. If $R$ therefore is low, the Gaussian curve is narrow, and the atoms behave more like a point charge, i.e., the curve in Fig. 9 flattens. If $R$ is nearly zero, each atom carries the same amount of charge, which equals to the total charge over the tube divided by the amount of atoms of the CNT. All points of the curve then align to a simple horizontal line.

On the other hand, if $R$ is quite large, the Gaussian curve flattens. That means that the areas in which the charges are spread overlap each other. This results in alternating charge values in each row of atoms as shown in Fig. 9a and $\mathrm{b}$ for $R=0.9 \AA$. This effect and therefore the maximal value of the numerically obtained charges increase with an increasing $R$.

The curve for $R=0.6863 \AA$ is indicated by the symbol “*”. This is the usual value for $R$ as used for the calculation of all test cases. Please note that this value is also used in the research by Keblinski [21], Mayer [24], and $\mathrm{Li}$ [22]. In their research, this was found to be a good agreement between ab initio calculations and the performed molecular mechanics simulations.

In Fig. 10, the influence of the bond length $d_{c-c}$-as mentioned in Sect. 2.1-is depicted. For a smaller bond length, the whole tube is shorter if the same configuration is used. Therefore, the distance between the 


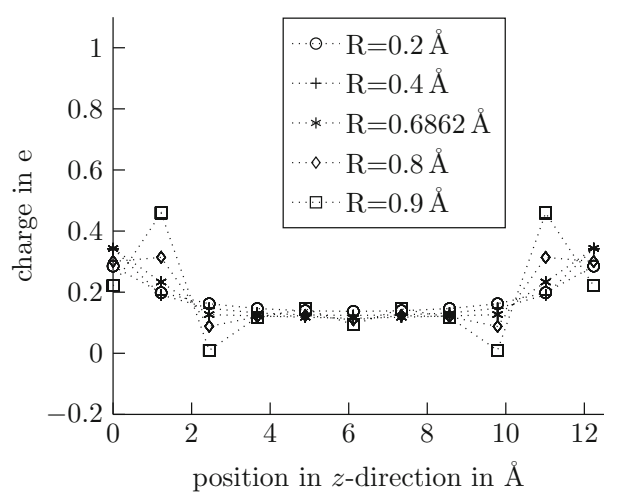

(a)

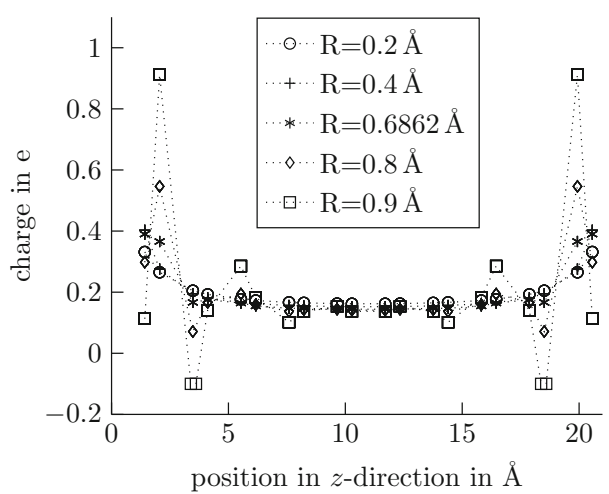

(b)

Fig. 9 a $(5,5)$ CNT; $\mathbf{b}(5,0)$ CNT with a total charge of 20e and 5 unit cells according to the model by Mayer. The values of the parameter $R$ are varied; $R=\{0.2,0.4,0.6862,0.8,0.9 \AA$

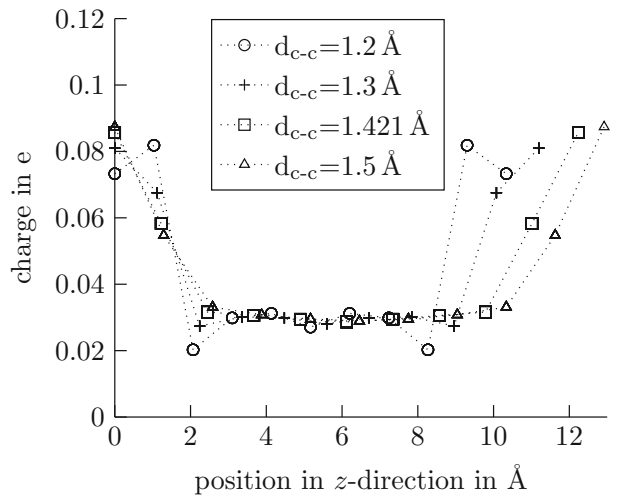

(a)

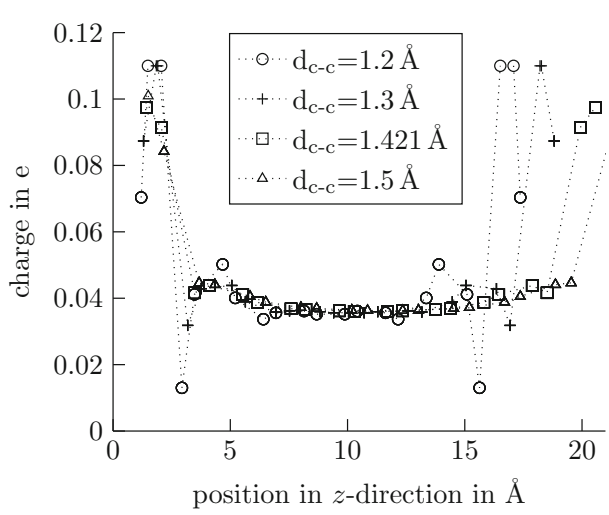

(b)

Fig. 10 a $(5,5)$ CNT; $\mathbf{b}(5,0)$ CNT with a total charge of $5 \mathrm{e}$ and 5 unit cells according to the model by Mayer. The values of the parameter $d_{c-c}$ are varied; $d_{c-c}=\{1.2,1.3,1.421,1.5 \AA\}$

rows of atoms is shorter. In the curves given in Fig. 10, the model by Mayer is used. Therefore, the interaction between the width of the Gaussian curve with the parameter $R$ and the bond length $d_{c-c}$ can be observed. As mentioned previously, $R$ describes how the charge belonging to one nucleus is distributed over the nanotube. If $R$ is fixed and the distances between rows of atoms is changed, the same effects as shown in Fig. 9 occur due to the ratio between $R$ and $d_{c-c}$. If $R$ is fixed at $R=0.6862 \AA$ and the bond length $d_{c-c}$ decreases, a similar behavior like in the case of a fixed distance and an increasing $R$ is observed. Therefore, if the bond length decreases, the charge begins to fluctuate as the area in which the charges are spread, overlaps. Please note, that — due to a better visibility — in Fig. 10b some data points are not displayed. On the other hand, with an increasing atomic distance the interaction declines. If the distance is further increased-which is not shown in order to improve the clarity of the graph, the charge distribution flattens like it does if $R$ is decreased. An overview of the numerical investigations (test case I) and the used parameters are given in Table 1.

\subsection{Test case II: applied external field}

In order to understand the electric behavior of the CNTs, not only the reaction due to an overall charge but also due to an applied electric field has to be investigated. This will be discussed in the present Section. With the knowledge of the electric properties, it is possible to create an electromechanical model of a carbon nanotube. This could lead to a more efficient construction for, e.g., actuators made out of CNTs. In the different investigations, electric fields of $E=0.1 \mathrm{~V} / \AA, 0.5 \mathrm{~V} / \AA ⿻, 1 \mathrm{~V} / \AA, 5 \mathrm{~V} / \AA$ and $10 \mathrm{~V} / \AA$ are applied to an $(5,5)$ armchair CNT and a $(5,0)$ zigzag nanotube with five unit cells, respectively. In the following, it is shown how 
Table 1 Parameters used for the numerical simulations of the test case I

\begin{tabular}{|c|c|c|c|c|c|c|c|c|}
\hline Figure & $n$ & $m$ & uc & $\begin{array}{l}d_{c-c} \\
\text { in } \AA\end{array}$ & $\begin{array}{l}R \\
\text { in } \AA\end{array}$ & $\begin{array}{l}\text { Electric field } \\
\text { in } V / \AA\end{array}$ & $\begin{array}{l}\text { Total charge } \\
\text { in e }\end{array}$ & Model \\
\hline 3(a) & 5 & 5 & 5 & 1.421 & 0.6862 & 0 & 5 & Mayer \\
\hline (b) & 5 & 0 & 5 & 1.421 & 0.6862 & 0 & 5 & Mayer \\
\hline 4(a) & 5 & 5 & 5 & 1.421 & 0.6862 & 0 & 20 & Mayer \\
\hline (b) & 5 & 0 & 5 & 1.421 & 0.6862 & 0 & 20 & Mayer \\
\hline $5(a)$ & 5 & 5 & 5 & 1.421 & 0.6862 & 0 & $1,2,5,10,20$ & Mayer \\
\hline (b) & 5 & 0 & 5 & 1.421 & 0.6862 & 0 & $1,2,5,10,20$ & Mayer \\
\hline 6(a) & 5 & 5 & 5 & 1.421 & 0.6862 & 0 & 20 & $\mathrm{Li}$ and Chou \\
\hline (b) & 5 & 0 & 5 & 1.421 & 0.6862 & 0 & 20 & $\mathrm{Li}$ and Chou \\
\hline 7 (a) & 5 & 5 & 5 & 1.421 & 0.6862 & 0 & 20 & Li and Chou; Mayer \\
\hline (b) & 5 & 0 & 5 & 1.421 & 0.6862 & 0 & 20 & Li and Chou; Mayer \\
\hline 8(a) & 5 & 5 & 20 & 1.421 & 0.6862 & 0 & 20 & Li and Chou; Mayer \\
\hline (b) & 5 & 0 & 20 & 1.421 & 0.6862 & 0 & 20 & Li and Chou; Mayer \\
\hline 9 (a) & 5 & 5 & 5 & 1.421 & $0.2,0.4,0.6862,0.8,0.9$ & 0 & 20 & Mayer \\
\hline (b) & 5 & 0 & 5 & 1.421 & $0.2,0.4,0.6862,0.8,0.9$ & 0 & 20 & Mayer \\
\hline 10 (a) & 5 & 5 & 5 & $1.2,1.3,1.421,1.5$ & 0.6862 & 0 & 5 & Mayer \\
\hline (b) & 5 & 0 & 5 & $1.2,1.3,1.421,1.5$ & 0.6862 & 0 & 5 & Mayer \\
\hline
\end{tabular}

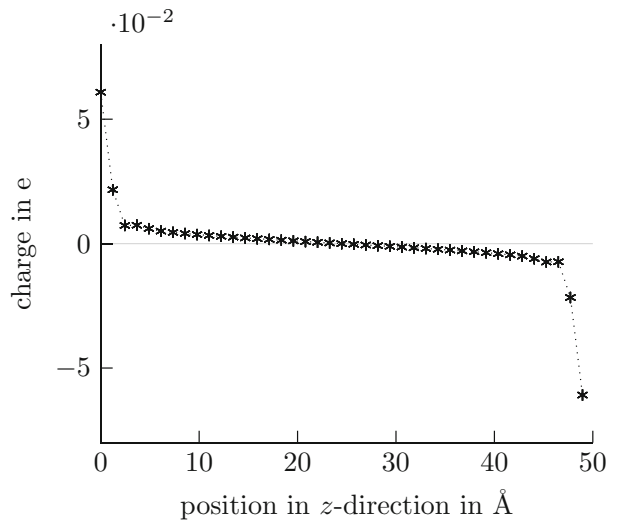

(a)

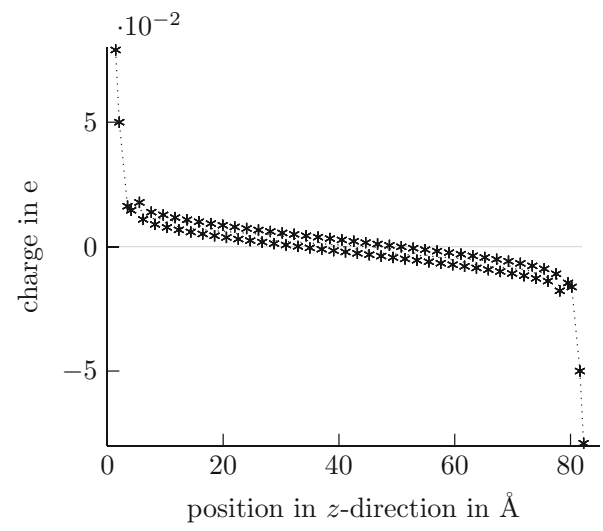

(b)

Fig. 11 a $(5,5)$ CNT; $\mathbf{b}(5,0)$ CNT in an electric field with $E=0.1 \mathrm{~V} / \AA$ and 20 unit cells according to the model by Mayer

the charges are distributed if the electric field strength of the applied external field increases. The parameters used for this test case (test case II) are given in Table 2.

In Fig. 11, the charge on each atom with respect to the position on the $z$-axis of a tube of 20 unit cells is shown. In Fig. 11a, an armchair tube and in Fig. 11b a zigzag CNT is shown. Both tubes have been charged with an ideal electric field of $E=0.1 \mathrm{~V} / \AA$ in (longitudinal) $z$-direction. In difference to Sect. 3.1, there is no overall external charge on the tube.

In Fig. 11a, the general behavior can be observed. As already shown in the simulations using a total charge, the values of the charges in the middle of the tube are not as high as at the ends. It can be seen that-due to the applied electric field - there is a slight decrease in the charge versus $z$. The last two rows of atoms behave differently. They show an increase in the charge values. All charges summed up equal to zero, as prescribed.

In Fig. 11b, the absolute values of the charges in the middle of the tube show an alternating behavior. The explanation for this can be found in the parameter $R$ describing the width of the Gaussian distribution which characterizes the interacting of the charges in the model by Mayer [24]. In Refs. [22,24], $R$ is stated for the armchair nanotubes but not for the zigzag tubes. All the simulations were performed with $R=0.6862 \AA$. It might be possible that a slightly lower value of $R$ would fit better for a zigzag CNT.

Next, the effect of different external electric fields is investigated. The results of the numerical simulation are shown in Fig. 12. A shorter tube is used consisting of only five unit cells. It is expected that the general behavior remains the same for a longer nanotube. If the electric field is larger, the values of the charges increase. They maintain the point symmetry around the exact middle of the tubes. As mentioned before, in Fig. 11(b) the values of the charge around the middle oscillate slightly, due to the influence of the parameter $R$. 


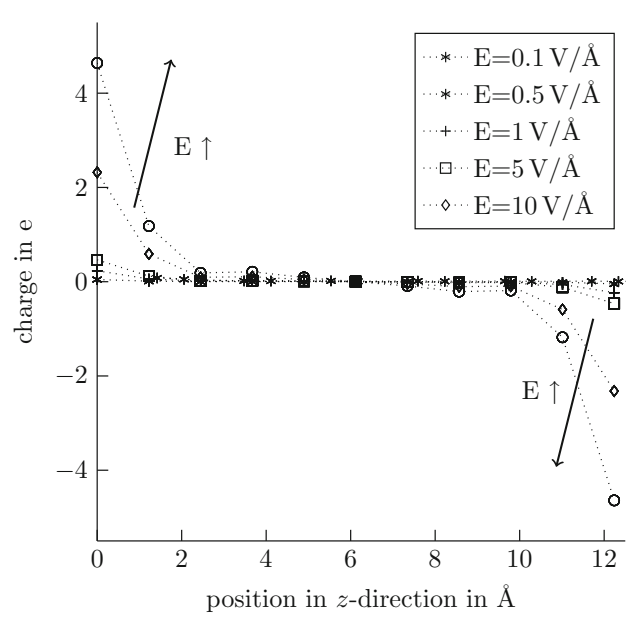

(a)

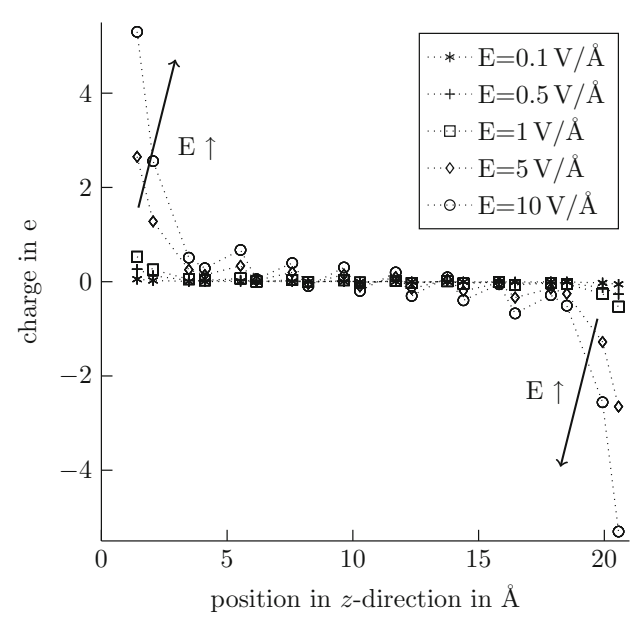

(b)

Fig. 12 a (5,5) CNT; b (5,0) CNT with a length of 5 unit cells subjected to different electric fields $(E=0.1,0.5,1,5,10 \mathrm{~V} / \AA ̊$ ) according to the model by Mayer

As already explained in Sect. 3.1, the reaction of the charges to the external field or to the total charge can be seen best in the last two rows of atoms toward the ends of the tubes. Figures 9 and 11 confirm that the length of the CNT does not affect this correlation.

\subsection{Test case III: overall charge and applied external field}

In most of the real applications, the factor of an overall charge and an applied external field cannot be considered completely separated. Therefore, in the last test case, both effects will be combined, in order to accomplish a complete impression of the electric behavior of armchair and zigzag nanotubes. First, the CNT is charged with an overall charge of $5 \mathrm{e}$. Then, an external electric field of $E=0.1 \mathrm{~V} / \AA$ in (longitudinal) $z$-direction is applied to the carbon nanotube. Again, like in Sect. 3.1 both, armchair and zigzag carbon nanotubes are investigated. Furthermore, the effect of increasing the overall charge combined with an applied external field will be discussed.

The parameters used for this test case (test case III) are given in Table 3.

The charge with respect to the position of the atoms of the CNT is depicted for armchair in Fig. 13a and for zigzag tubes in Fig. 13b. According to the total charge of $5 \mathrm{e}$, the atoms in the middle region are charged similarly as in Fig. 3. A larger difference can be observed at both ends of the nanotubes. It can be seen that the effects of the external field and of the total charge are combined. At the left end of the tubes, both influences are summed up. On the right side, the contributions work against each other. The combination of a decreased charge due to the electric field and an increase in the charge due to the applied overall charge tends to result in lower values of charges at the ends of the tube.

In the next step, the external electric field is kept constant $E=0.1 \mathrm{~V} / \AA$ and the overall charge on the tubes varies, see Fig. 14. As expected, the same behavior like in Fig. 5 occurs, with the additional effect depicted in Fig. 13.

Table 2 Parameters used for the numerical simulations of the test case II

\begin{tabular}{llllllllr}
\hline Figure & $n$ & $m$ & uc & $\begin{array}{l}d_{c-c} \\
\text { in } \AA\end{array}$ & $\begin{array}{l}R \\
\text { in } \AA\end{array}$ & $\begin{array}{l}\text { Electric field } \\
\text { in V/A }\end{array}$ & $\begin{array}{l}\text { Total charge } \\
\text { in e }\end{array}$ & Model \\
\hline Figure 11a & 5 & 5 & 20 & 1.421 & 0.6862 & 0.1 & 0 & Mayer \\
Figure 11b & 5 & 0 & 20 & 1.421 & 0.6862 & 0.1 & 0 & Mayer \\
Figure 12a & 5 & 5 & 5 & 1.421 & 0.6862 & $0.1,0.5,1,5,10$ & 0 & Mayer \\
Figure 12b & 5 & 0 & 5 & 1.421 & 0.6862 & $0.1,0.5,1,5,10$ & 0 & Mayer \\
\hline
\end{tabular}




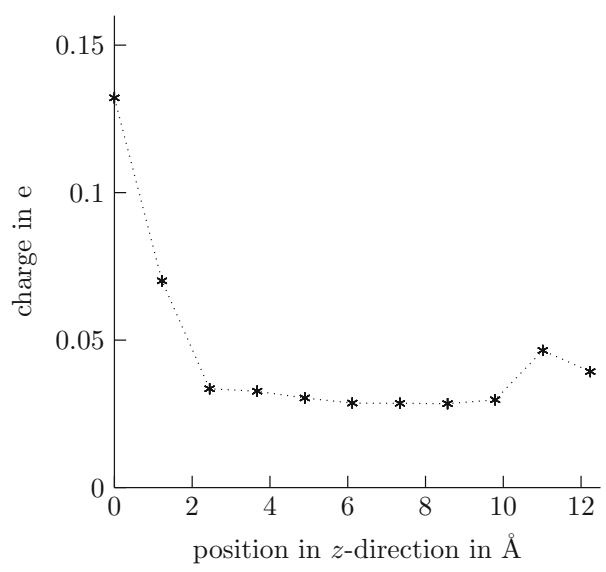

(a)

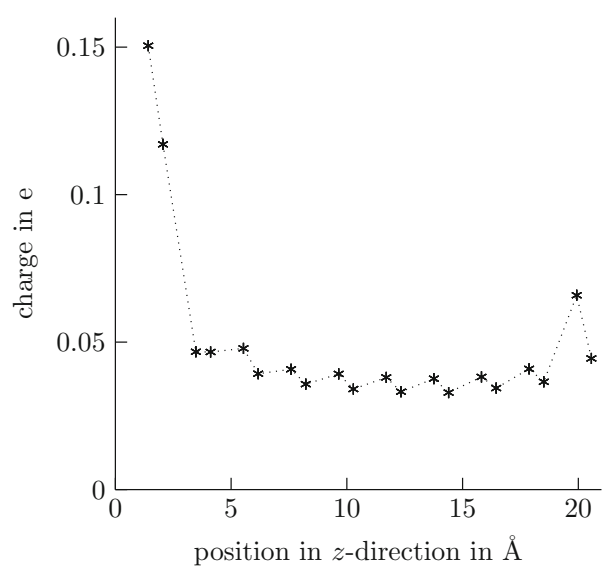

(b)

Fig. 13 a $(5,5)$ CNT; b $(5,0)$ CNT in an electric field with $E=0.1 \mathrm{~V} / \AA ̊$, with a total charge of 5 e and a length of 5 unit cells according to the model by Mayer

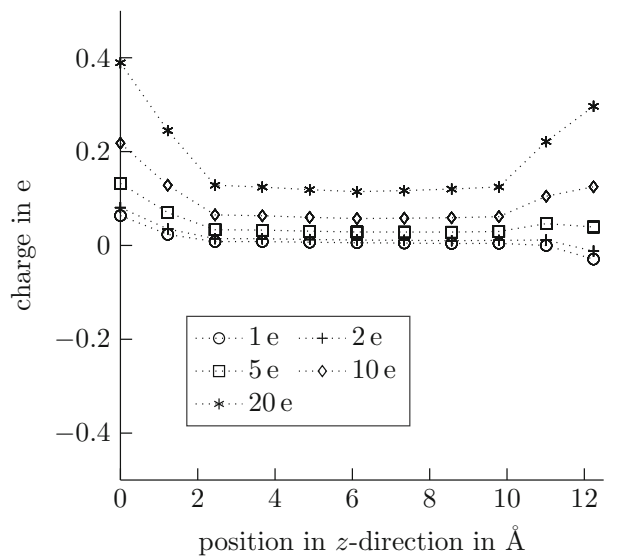

(a)

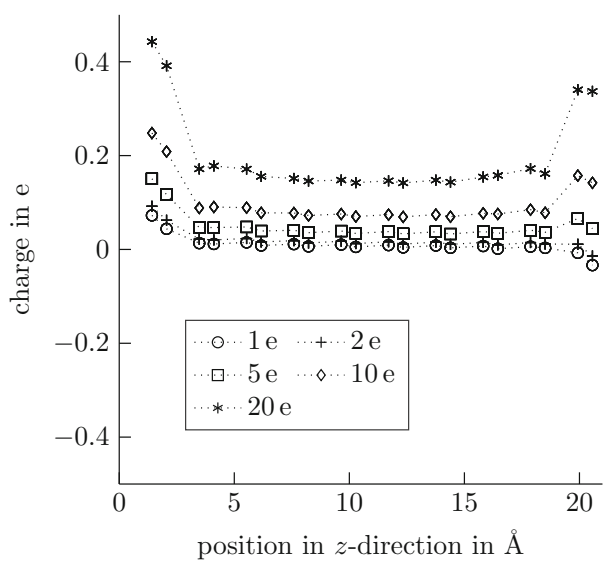

(b)

Fig. 14 a (5,5) CNT; b (5,0) CNT in an electric field with $E=0.1 \mathrm{~V} / \AA$, with different total charges of 1e, 2e, 5e, 10e, and 20e, and a length of 5 unit cells according to the model by Mayer

Table 3 Parameters used for the numerical simulations of the test case III

\begin{tabular}{lllllllll}
\hline Figure & $n$ & $m$ & uc & $\begin{array}{l}d_{c-c} \\
\text { in } \AA\end{array}$ & $\begin{array}{l}R \\
\text { in } \AA\end{array}$ & $\begin{array}{l}\text { Electric field } \\
\text { in V/A }\end{array}$ & $\begin{array}{l}\text { Total charge } \\
\text { in e }\end{array}$ & Model \\
\hline Figure 13a & 5 & 5 & 5 & 1.421 & 0.6862 & 0.1 & 5 & Mayer \\
Figure 13b & 5 & 0 & 5 & 1.421 & 0.6862 & 0.1 & 5 & Mayer \\
Figure 14a & 5 & 5 & 5 & 1.421 & 0.6862 & 0.1 & $1,2,5,10,20$ & Mayer \\
Figure 14b & 5 & 0 & 5 & 1.421 & 0.6862 & 0.1 & $1,2,5,10,20$ & Mayer \\
\hline
\end{tabular}

\subsection{Preliminary assessment of the test cases with respect to electromechanical coupling}

In test case I, the nanotubes were charged with an overall charge. Therefore, atoms at the same Z-position have the same value and polarity of charge. Since the same charges repel each other, it is expected that the nanotube will expand radially. Additionally, the tube will expand along the $z$-axis, due to the distributed charges. Due to the higher charges, and therefore the higher repulsive forces, the extensions at both ends of the CNT are expected to be larger than in the middle region. This extension of the nanotube will again lead to a redistribution of the charges, i.e., especially at the ends of the CNT, the charge accumulation is expected to be reduced, due to the larger resulting atomic distances. 
In the last test case, an electric field was applied to an already charged nanotube. As mentioned before, the nanotube expands radially due to the same polarity of the charges at each atom of the same $z$-position. Also in each half of the tube the charges are polarized in the same way; therefore, they react repulsive, and the tube will get longer. For a high electric field strength, both ends of the tube are charged in the opposite way resulting in an attractive effect. This could compress or deform the tube.

In further studies, it will be investigated which of both effects-repelling or attracting-are stronger. Therefore, a coupled electromechanical formulation will be derived and studied intensively.

\section{Conclusions}

In the present research, a systematic analysis of the behavior of charged carbon nanotubes exposed to electric fields is performed. Two different modeling approaches [22,24] are compared with each other. The main focus of this work is the reaction of armchair and zigzag CNTs: (i) to an applied overall charge, (ii) to an applied external electric field, and (iii) to the combination of both effects. Additionally, the effect of the carbon bond length and the effect of the width $R$ of the Gaussian distribution in which the charge on the nucleus is spread is investigated. Concluding, the present work investigates the charge distribution over the tubes.

An applied overall total charge of the nanotube shows that the charge is symmetrically distributed on the CNT, independent of the tube configuration. The highest values of charge can be found at the ends of the nanotube. Even if the CNT is constructed by a large number of unit cells, i.e., it is quite long, only the last few atoms are higher charged.

This research compares two different modeling approaches, the model by $\mathrm{Li}$ and Chou $[22,33]$ and the model by Mayer [24]. Both models give similar results with respect to the overall behavior, but there are significant differences in the values of the charges at the nanotube ends. The model by $\mathrm{Li}$ and Chou shows a more extreme step to higher values of the charge at the last row of atoms of the tube. The model by Mayer is more homogeneous, i.e., the increase is not as high as in the model by $\mathrm{Li}$ and Chou.

It is also observed that the choice of the width of the Gaussian distribution $R$ in the model by Mayer [24] is important. If it is too low, the behavior is similar to point charges. If $R$ is too high, the charge is distributed quite evenly all over the nanotube, i.e., the charges interact largely with each other, and also oscillations of the charge occur. Besides, the parameter $R$ has to be adjusted to the length of the carbon bond $d_{c-c}$ and to the configuration of the CNT for both, armchair and zigzag tubes. In the present research, $R=0.6862 \AA$ is used, according to other investigations available in the literature. This value fits very well for armchair nanotubes. For a zigzag configuration, a smaller value of $R$ should be chosen in order to eliminate the small oscillations of the charge.

If an electric field is applied, the charges are distributed point symmetrically with respect to the middle cross-sectional area of the nanotube. The effect of an overall charge and an electric field superpose each other if both are applied.

In further studies, the electromechanical coupling, i.e., the deformation of the tube due to the charge distribution, will be investigated. By applying an electromechanical model, e.g., for nanotweezers, the superposition of attracting and repelling effects, leading to deformation (expansion, compression or bending) as a consequence of the applied charge and the applied electric field, will be investigated.

Acknowledgements The support of the German Science Foundation (DFG) within the Grant WA2323/6-2 is gratefully acknowledged.

Funding Open Access funding enabled and organized by Projekt DEAL.

Open Access This article is licensed under a Creative Commons Attribution 4.0 International License, which permits use, sharing, adaptation, distribution and reproduction in any medium or format, as long as you give appropriate credit to the original author(s) and the source, provide a link to the Creative Commons licence, and indicate if changes were made. The images or other third party material in this article are included in the article's Creative Commons licence, unless indicated otherwise in a credit line to the material. If material is not included in the article's Creative Commons licence and your intended use is not permitted by statutory regulation or exceeds the permitted use, you will need to obtain permission directly from the copyright holder. To view a copy of this licence, visit http://creativecommons.org/licenses/by/4.0/. 


\section{References}

1. Radushkevich, L., Lukyanovich, V.: About the structure of carbon formed by thermal decomposition of carbon monoxide on iron substrate. J. Phys. Chem. (Moscow) 26, 88-95 (1952)

2. Iijima, S.: Helical microtubules of graphitic carbon. Nature 354, 56-58 (1991)

3. Lu, W., Zu, M., Byun, J.-H., Kim, B.-S., Chou, T.-W.: State of the art of carbon nanotube fibers: opportunities and challenges. Adv. Mater. 24, 1805-1833 (2012)

4. Dresselhaus, M., Dresselhaus, G., Saito, R.: Physics of carbon nanotubes. Carbon 33, 883-891 (1995)

5. Dresselhaus, G., Dresselhaus, M.S., Saito, R.: Physical Properties of Carbon Nanotubes. World Scientific, Singapore (1998)

6. Yakobson, B. I., Avouris, P.: Mechanical properties of carbon nanotubes, In: Dresselhaus, M.S., Dresselhaus, G., Avouris, Ph. (eds.) Carbon Nanotubes, Springer, pp. 287-327 (2001)

7. Kong, J., Franklin, N.R., Zhou, C., Chapline, M.G., Peng, S., Cho, K., Dai, H.: Nanotube molecular wires as chemical sensors. Science 287, 622-625 (2000)

8. Collins, P.G., Bradley, K., Ishigami, M., Zettl, dA.: Extreme oxygen sensitivity of electronic properties of carbon nanotubes. Science 287, 1801-1804 (2000)

9. Chopra, S., McGuire, K., Gothard, N., Rao, A., Pham, A.: Selective gas detection using a carbon nanotube sensor. Appl. Phys. Lett. 83, 2280-2282 (2003)

10. Zaporotskova, I.V., Boroznina, N.P., Parkhomenko, Y.N., Kozhitov, L.V.: Carbon nanotubes: sensor properties. A review. Modern Electron. Mater. 2, 95-105 (2016)

11. Dresselhaus, M.S., Dresselhaus, G., Eklund, P.C.: Science of Fullerenes and Carbon Nanotubes: Their Properties and Applications. Elsevier, Amsterdam (1996)

12. Louie, S. G.: Electronic properties, junctions, and defects of carbon nanotubes, In: Dresselhaus, M.S., Dresselhaus, G., Avouris, Ph. (eds.) Carbon Nanotubes, Springer pp. 113-145 (2001)

13. Avery, A.D., Zhou, B.H., Lee, J., Lee, E.-S., Miller, E.M., Ihly, R., Wesenberg, D., Mistry, K.S., Guillot, S.L., Zink, B.L., et al:: Tailored semiconducting carbon nanotube networks with enhanced thermoelectric properties. Nature Energy 1, 1-9 (2016)

14. Kim, P., Lieber, C.M.: Nanotube Nanotweezers. Science 286, 2148-2150 (1999)

15. Akita, S., Nakayama, Y., Mizooka, S., Takano, Y., Okawa, T., Miyatake, Y., Yamanaka, S., Tsuji, M., Nosaka, T.: Nanotweezers consisting of carbon nanotubes operating in an atomic force microscope. Appl. Phys. Lett. 79, 1691-1693 (2001)

16. Baughman, R.H., Cui, C., Zakhidov, A.A., Iqbal, Z., Barisci, J.N., Spinks, G.M., Wallace, G.G., Mazzoldi, A., De Rossi, D., Rinzler, A.G., et al.: Carbon nanotube actuators. Science 284, 1340-1344 (1999)

17. Kosidlo, U., Omastová, M., Micusík, M., Ćirić-Marjanović, G., Randriamahazaka, H., Wallmersperger, T., Aabloo, A., Kolaric, I., Bauernhansl, T.: Nanocarbon based ionic actuators-a review. Smart Mater. Struct. 22, 104022 (2013)

18. Tans, S.J., Verschueren, A.R., Dekker, C.: Room-temperature transistor based on a single carbon nanotube. Nature 393, 49-52 (1998)

19. Hills, G., Lau, C., Wright, A., Fuller, S., Bishop, M.D., Srimani, T., Kanhaiya, P., Ho, R., Amer, A., Stein, Y., et al.: Modern microprocessor built from complementary carbon nanotube transistors. Nature 572, 595-602 (2019)

20. Peng, Z., Ng, A.L., Kwon, H., Wang, P., Chen, C.-F., Lee, C.S., Wang, Y.: Graphene as a functional layer for semiconducting carbon nanotube transistor sensors. Carbon 125, 49-55 (2017)

21. Keblinski, P., Nayak, S.K., Zapol, P., Ajayan, P.M.: Charge distribution and stability of charged carbon nanotubes. Phys. Rev. Lett. 89, 255503 (2002)

22. Li, C., Chou, T.-W.: Electrostatic charge distribution on single-walled carbon nanotubes. Appl. Phys. Lett. 89, 063103 (2006)

23. Wang, Z., Devel, M.: Electrostatic deflections of cantilevered metallic carbon nanotubes via charge-dipole model. Phys. Rev. B 76, 195434 (2007)

24. Mayer, A.: Formulation in terms of normalized propagators of a charge-dipole model enabling the calculation of the polarization properties of fullerenes and carbon nanotubes. Phys. Rev. B 75, 045407 (2007)

25. Eberhardt, O., Wallmersperger, T.: Mechanical properties and deformation behavior of carbon nanotubes calculated by a molecular mechanics approach. Smart Struct. Syst. 13, 685-709 (2014)

26. Cox, B.J., Hill, J.M.: Exact and approximate geometric parameters for carbon nanotubes incorporating curvature. Carbon 45, 1453-1462 (2007)

27. Rotkin, S.V., Shrivastava, V., Bulashevich, K.A., Aluru, N.: Atomistic capacitance of a nanotube electromechanical device. Int. J. Nanosci. 1, 337-346 (2002)

28. Bulashevich, K.A., Rotkin, S.: Nanotube devices: a microscopic model. J. Exp. Theor. Phys. Lett. 75, 205-209 (2002)

29. Ke, C., Espinosa, H.D., Pugno, N.: Numerical analysis of nanotube based nems devices-part ii: Role of finite kinematics, stretching and charge concentrations. J. Appl. Mech. 72(5), 726-731 (2005)

30. Li, C.-Y., Chou, T.-W.: Charge-induced strains in single-walled carbon nanotubes. Nanotechnology 17, 4624-4628 (2006)

31. Eberhardt, O., Wallmersperger, T.: Energy consistent modified molecular structural mechanics model for the determination of the elastic properties of single wall carbon nanotubes. Carbon 95, 166-180 (2015)

32. Eberhardt, O., Wallmersperger, T.: Advanced molecular structural mechanics model for carbon nanotubes incorporating the 2nd generation rebo potential. Int. J. Eng. Sci. 144, 103137 (2019)

33. Li, C., Chou, T.-W.: Theoretical studies on the charge-induced failure of single-walled carbon nanotubes. Carbon 45, 922-930 (2007)

34. Mayer, A., Åstrand, P.-O.: A charge-dipole model for the static polarizability of nanostructures including aliphatic, olephinic, and aromatic systems. J. Phys. Chem. A 112, 1277-1285 (2008)

35. Allred, A.L.: Electronegativity values from thermochemical data. J. Inorganic Nuclear Chem. 17, 215-221 (1961)

36. Mulliken, R.S.: A new electroaffinity scale; together with data on valence states and on valence ionization potentials and electron affinities. J. Chem. Phys. 2, 782-793 (1934) 
37. Bultinck, P., Langenaeker, W., Lahorte, P., De Proft, F., Geerlings, P., Waroquier, M., Tollenaere, J.P.: The electronegativity equalization method i: Parametrization and validation for atomic charge calculations. J. Phys. Chem. A 106, 7887-7894 (2002)

Publisher's Note Springer Nature remains neutral with regard to jurisdictional claims in published maps and institutional affiliations. 